OPEN ACCESS

Edited by:

Eric Merkley,

University of Toronto, Canada

Reviewed by:

Clifton Van Der Linden, McMaster University, Canada

Stephen Utych,

Boise State University, United States

*Correspondence:

Matt Motta

matthew.motta@okstate.edu

Specialty section:

This article was submitted to

Politics of Technology,

a section of the journal

Frontiers in Political Science

Received: 16 November 2020 Accepted: 04 January 2021

Published: 28 January 2021

Citation:

Motta M, Sylvester S, Callaghan T and Lunz-Trujillo K (2021) Encouraging COVID-19 Vaccine Uptake Through Effective Health Communication.

Front. Polit. Sci. 3:630133.

doi: 10.3389/fpos.2021.630133

\section{Encouraging COVID-19 Vaccine Uptake Through Effective Health Communication}

\author{
Matt Motta $^{1 *}$, Steven Sylvester ${ }^{2}$, Timothy Callaghan ${ }^{3}$ and Kristin Lunz-Trujillo ${ }^{4}$ \\ ${ }^{1}$ Department of Political Science, Oklahoma State University, Stillwater, OA, United States, ${ }^{2}$ History \& Political Science \\ Department, Utah Valley University, Orem, UT, United States, ${ }^{3}$ Department of Health Policy and Management, Texas A and M \\ University, College Station, TX, United States, ${ }^{4}$ Deaprtment of Political Science, Carleton College, Northfield, MN, United States
}

Context: Overcoming the COVID-19 pandemic will require most Americans to vaccinate against the virus. Unfortunately, previous research suggests that many Americans plan to refuse a vaccine; thereby jeopardizing collective immunity. We investigate the effectiveness of three different health communication frames hypothesized to increase vaccine intention; emphasizing either 1) personal health risks, 2) economic costs, or 3) collective public health consequences of not vaccinating.

Methods: In a large $(N=7,064)$ and demographically representative survey experiment, we randomly assigned respondents to read pro-vaccine communication materials featuring one of the frames listed above. We also randomly varied the message source (ordinary people vs. medical experts) and availability of information designed the "prebunk" potential misinformation about expedited clinical trial safety.

Findings: We find that messages emphasizing the personal health risks and collective health consequences of not vaccinating significantly increase Americans' intentions to vaccinate. These effects are similar in magnitude irrespective of message source, and the inclusion of pre-bunking information. Surprisingly, economic cost frames have no discernible effect on vaccine intention. Additionally, despite sharp partisan polarization in public vaccination intentions, we find that these effects are no different for Democrats, Republicans, and Independents alike.

Conclusion: Health communicators hoping to encourage vaccination may be effective by appealing to the use personal and collective health risks of not vaccinating.

Keywords: COVID-19, misinformation, health communciation, political psychology, vaccine skepticism, public opinion, public health, COVID-19 vaccine

\section{INTRODUCTION}

Ending the SARS-CoV-2 (COVID-19) pandemic will require unprecedented collective action, on a global, national, and sub-national scale. In addition to social distancing and practicing other prosocial health behaviors (e.g., wearing masks in public places), hundreds of millions of Americans must choose to receive a COVID-19 vaccine, once it becomes widely available. By some estimates, up to 70 percent of Americans may need to develop antibodies to the disease -- either through contracting (and recovering from) the disease and/or through inoculation -- in order to put the virus' spread into decline (Bartsch et al., 2020; Britton et al.,2020; Kwok et al., 2020). 
Many Americans, however, may refuse a vaccine for COVID19 once it becomes available. According to recent public opinion research, somewhere between one fifth and one third of Americans do not plan to vaccinate, and many more remain unsure (Callaghan et al., 2020; Thigpen and Funk, 2020). This puts the nation in jeopardy of failing to hit herd immunity thresholds. Consequently, devising health communication strategies that effectively encourage Americans to vaccinate against COVID-19 could have critically important implications for public health. The effectiveness of pro-vaccine health communication messaging tactics could play a key role in determining how quickly the United States is able to resume life "as normal." What these messages might look like, however, and whether or not they will be effective is an open question.

In this paper, we report the results of a novel survey experiment -- embedded in a large and demographically representative survey $(\mathrm{N}=7,064)$-- which tests the effectiveness of three pro-vaccine messaging tactics ("frames"). Based on previous insights from social psychology, health communication, and political science research, we suspect that Americans will be more likely to vaccinate if they are presented with information highlighting the personal health risks (i.e., the possibility of getting seriously sick), economic costs (i.e., the financial burdens associated with the economy "shutting down" in order to contain the virus' spread), and/or the collective public health consequences (i.e., the possibility of infecting others; including vulnerable populations) of failing to vaccinate.

Additionally, we recognize that these messages -- if and when they are implemented outside of the controlled survey environment -- are likely to vary in both communication source (e.g., whether messages originate from medical experts vs. lay sources) and the amount of information they provide about the process of determining vaccine safety and efficacy via clinical trials (i.e., whether or not they preempt or "pre-bunk" concerns that a vaccine developed in an accelerated timeframe will not be safe and effective). Consequently, we provide a series of exploratory tests investigating whether or not certain sources are more effective at communicating each of the aforementioned frames than others, and whether or not information about the rigors of clinical trials might also increase message receptivity.

Consistent with our theoretical expectations, we find that messages highlighting the personal health risks and collective public health consequences of failing to vaccinate positively and significantly increase vaccination intentions. Exploratory analyses reveal little evidence that the source and/or presence (vs. absence) of clinical trial information influences the effectiveness of these frames. Interestingly, however, we find that economic loss frames are ineffective at moving vaccine intention.

Further, we report preliminary evidence suggesting that, while personal and collective health risk frames are effective at increasing vaccination intentions in the general public, they have (at best) a limited influence in doing the same for those already skeptical about the vaccine's eventual safety and efficacy. This suggests that efforts to influence skeptics' receptiveness to vaccination, ought to consider new and stronger efforts to highlight the vaccine's safety and efficacy.
Our results offer a potential path forward for health communicators hoping to devise messaging strategies aimed at increasing vaccine uptake. In general, our results recommend focusing on either the personal or community health risks of the failure to vaccinate; noting that the latter may be particularly effective at increasing intention among those least likely to vaccinate. Additionally, while messages originating from expert sources are not necessarily less effective, our results consistently document that messages from ordinary Americans' are effective at increasing intended vaccine uptake via these two messaging strategies.

Moreover, in addition to the practical health communication applications of our research, our work substantively advances previous scholarly research on pro-vaccine messaging. Typically, scholars study the efficacy of communication strategies that encourage vaccination against illnesses that have existed for many years, such as childhood vaccines for the measles or vaccines against seasonal influenza. Whether or not insights from previous studies hold amid rapidly changing pandemic conditions, and in response to this particular and novel public health threat, is an open question. Support for our theoretical expectations may help further highlight the generalizability of results from previous social science research. We conclude by discussing how government, non-profit, and other actors might co-opt these lessons into future efforts to increase public willingness to vaccinate against COVID-19.

\section{THE COVID-19 PANDEMIC AND VACCINE HESITANCY}

Since its initial introduction to the human population in Wuhan China in 2019, the COVID-19 pandemic has imposed considerable health, economic, and social costs on populations across the globe. By early August in 2020, over eighteen million people worldwide had been confirmed to have contracted the virus, with over 600,000 attributed fatalities in the first six months after widespread transmission (COVID-19 Dashboard, 2020). The United States has faced a disproportionate share of this burden with the most confirmed cases worldwide, and the Centers for Disease Control and Prevention estimating that actual case counts could be somewhere between 6-24 times higher than the confirmed count (Havers et al., 2020).

To combat the growing pandemic, public health officials around the globe have pursued health communication and mitigation strategies to slow the spread of the disease. These efforts have included educating individuals about appropriate hand washing, social distancing, and the symptoms of COVID19 , as well as pressuring politicians at all levels of government to mandate that individuals wear masks (CDC Centers for Disease Control and Prevention, 2020). Critically, however, even with these efforts in place, the disease continues to spread. For that reason, many scholars have suggested that the virus is likely to continue to spread-with disastrous consequences for human health and the economy-until herd immunity is reached through either widespread infection or widespread inoculation 
with a novel COVID-19 vaccine (Britton et al., 2020; Callaghan et al., 2020; Kwok et al., 2020).

Perhaps unsurprisingly then, there has been considerable global interest in the development and worldwide dissemination of a successful COVID-19 vaccine. As of July 21, 2020, there were 24 vaccine candidates in clinical evaluation and 142 candidates in preclinical evaluation (WHO World Health Organization, 2020). Despite this considerable effort by the scientific community, however, there is growing concern in the United States that COVID-19 vaccine hesitancy might jeopardize reaching the levels of vaccination needed to achieve herd immunity and end the pandemic. Estimates suggest that somewhere between 40 to 70 percent of Americans will need to develop antibodies to the disease-ideally through a COVID-19 vaccine (Bartsch et al., 2020; Britton et al., 2020; Kwok et al., 2020). Yet, mounting evidence suggests that up to half of Americans either do not intend to pursue a COVID-19 vaccine or are not yet sure about their vaccination intention (Callaghan et al., 2020; Cornwall, 2020).

While research on COVID-19 vaccine hesitancy is in its infancy, early work suggests that COVID-19 vaccine hesitancy is related to but distinct from hesitancy towards other vaccines. Individuals who endorse the anti-vaccine label or who are less trusting of the safety of vaccines generally are more likely to refuse an eventual COVID-19 vaccine (Lunz Trujillo and Motta, 2020). Notably however, large segments of the United States population who are otherwise trusting of vaccines are also hesitant about the COVID-19 vaccine (Lunz Trujillo and Motta, 2020).

COVID-19 vaccine hesitancy is driven by several factors, including concerns about the safety and efficacy of a vaccine developed with unprecedented speed, imprecise messaging from the Trump administration (i.e. Operation Warp Speed), and continual efforts by anti-vaccine advocates to sow doubt in the general public (Cohen, 2020; Hastline, 2020). To this point, research suggests that COVID-19 vaccine hesitancy is highest in the United States among Blacks, women, and conservatives. Conversely, those who place more trust in experts and have been tested for COVID-19 are less likely to be vaccine hesitant (Callaghan et al., 2020).

\section{OVERCOMING VACCINE HESITANCY THROUGH EFFECTIVE HEALTH COMMUNICATION}

\section{How Personal, Economic, and Collective Health Risk Frames Could Influence Vaccine Compliance}

Overcoming widespread COVID-19 vaccine hesitancy will take a concerted public health messaging campaign that encourages hesitant individuals to vaccine. Past science communication and vaccine hesitancy research offers useful guidance as we work towards identifying effective health messages to increase COVID-19 vaccination intention.

For example, research suggests that the presentation of vaccine safety information from the $\mathrm{CDC}$ can reduce misinformation about vaccination (Nyhan and Reifler, 2015; Vraga and Bode, 2017). Other research suggests that highlighting medical consensus about vaccine safety can also be effective at reducing public concern about vaccination, decreasing belief in misinformation about a link between childhood vaccines and autism, and increasing public support for vaccination (Van der Linden et al., 2015).

Existing research also suggests that efforts to change the attitudes and behaviors of skeptics are more likely to be successful when they recognize and validate the concerns of the individuals that persuasion attempts are aimed at (Kahan, 2010). Demonstrative of this point, recent research suggests that tailored communication strategies can be particularly effective in moving the vaccine hesitant. Specifically, presenting the public with tailored pro-vaccine messages that appeal to (rather than attempt to debunk) psychological aversions to vaccination can be effective at reducing vaccine misinformation (Lunz Trujillo et al., 2020). In the case of COVID-19, this would suggest that efforts to improve vaccination intention may be most successful when they focus not only on the science of vaccine safety and efficacy, but on framing the importance of vaccination in response to issues that Americans think about on a regular basis. In what follows, we consider the potential effectiveness of pro-vaccine messages that appeal to the personal, collective, and economic risks associated with the novel coronavirus.

First, personal risk is a factor that may encourage many Americans to vaccinate, even those who are typically vaccine hesitant. COVID-19 is a highly contagious virus linked with a number of adverse outcomes, including hospitalization, pneumonia, blood clots, septic shock, lung and organ damage, heart failure, acute respiratory failure, and death, among others (Cascella et al., 2020; Long et al., 2020). In the United States, COVID-19 is currently the third leading cause of death, behind only heart disease and cancer (Bean, 2020). Notably, Americans are aware of the adverse effects of COVID-19, and a majority are worried that they will contract the virus (Newport, 2020). Many fear contracting the disease and experiencing negative medical consequences, such as hospitalization or death, particularly for those who are older, have co-morbidities, or work in a profession where COVID-19 exposure risk is higher (Adams and Walls, 2020; Taylor et al., 2020).

Consequently, vaccination messages highlighting the personal health risks associated with the novel coronavirus may encourage vaccine uptake. Personal risk frames are common health communication strategies used to promote healthy behaviors, such as smoking cessation. These strategies are rooted in fears over harm and death. Terror Management Theory (TMT) argues that people engage in predictable psychological coping mechanisms when mortality is salient, i.e., when people are aware that death is inevitable and can happen at any time (Becker, 1973; Greenberg et al., 1986). Existing research finds that highlighting the potential for death or harm in oneself and others will push people to adopt healthier attitudes and behaviors when the trigger (in our case: COVID-19) is consciously linked with the threat of death or bodily harm (e.g., Arndt and Goldenberg, 2017; Haglin et al., 2019). 
Therefore, we expect that a frame highlighting personal risk should be effective in bolstering intention to receive the COVID19 vaccine:

\section{H1: Exposure to messages highlighting personal risk of failing to vaccinate against COVID-19 will increase the intention to vaccinate against COVID-19, on average, compared to exposure to a control message.}

Similarly, we also expect that frames highlighting how a COVID-19 vaccine will minimize risk to the collective health of the population will be effective in increasing people's intention to vaccinate. This is particularly true given that the serious complications related to contracting COVID-19 disproportionately affect certain at-risk subsets of the population. Members of the public who are not part of these groups may feel less motivation to vaccinate against COVID-19. Therefore, vaccination uptake may also depend on eliciting pro-social sentiments in the general public. A previous study encouraging influenza vaccination found that individuals exposed to pro-social messages-in the form of describing potential flu victims who could have serious adverse effects if others do not vaccinate-were more likely to intend to vaccinate; , including those who had previously refused to vaccinate (Li et al., 2016). Similarly, those who are more self-transcendent (more likely to put others' needs before their own) are more likely to engage in social distancing measures to prevent the spread of COVID-19 (Motta and Goren, 2020; see also; Van der Linden et al., 2020).

Consequently, engaging people's sympathies to think about the consequences of failing to vaccinate as a risk to vulnerable populations may be effective in shifting COVID-19 vaccination intention:

\section{H2: Exposure to messages highlighting the collective health consequences of failing to vaccinate against COVID-19 will increase the intention to vaccinate against COVID-19, on average, compared to exposure to a control message.}

Finally, we expect that highlighting the economic risks of failing to receive a COVID-19 vaccine will be effective in increasing people's intention to vaccinate. The economic impact of COVID-19 has been devastating, with millions of Americans forced into unemployed at rates surpassing the Great Recession and leaving many states with little choice but to pause reopening the economy due to a recent surge of the virus (Kochar, 2020; Reimann, 2020).

Most behavioral health research on vaccinations has focused on providing positive economic incentives to entice individuals to vaccinate. For example, research suggests that economic incentives can be a powerful motivator for vaccination behavior, pushing the hesitant and complacent towards vaccinating (Betsch et al., 2015; Bohm et al., 2016). Similarly, in a review, Kane et al. (2004) found that incentives (e.g., cash payments, gifts, lotteries) can influence behavior when the tasks participant are asked to complete are simple (i.e. immunization) versus more complex like maintaining a diet. Although less work has focused on the effects of economic loss on vaccine uptake, insights from Prospect Theory-i.e., the idea that people tend to place higher value on losses compared to equally sized gains (see: Kahneman, 2011 for a review)-lead us to suspect that insights gleaned from research on positive incentives also apply to negative economic outcomes. Consequently, we expect that emphasizing link between a failure to vaccinate and an individual's economic security (e.g., being able to go back to work, stimulating the macro-economy) rather than an economic incentive message could encourage individuals who are hesitant to receive a COVID vaccine to get one when one becomes available.

H3: Exposure to messages highlighting the economic risks of failing to vaccinate against COVID-19 will increase the intention to vaccinate against COVID-19, on average, compared to a control message.

Taken together, existing research offer several possible paths forward for reducing COVID-19 vaccine hesitancy. Our analysis serves as a first investigation of some of these strategies in the context of the pandemic-studying the potential influence of frames that highlight either personal health risks, the collective health risks, or the economic imperatives of vaccinating.

\section{How the Source of Pro-vaccine Messages Could Influence Compliance}

In addition to the aforementioned differences in provaccination arguments (frames), we recognize that messages are also likely to vary in their source (i.e., who is responsible for communicating the message). Doctors and other medical experts may seem like intuitive and potentially effective communicators for messages emphasizing the medical and broader social benefits of a COVID-19 vaccine. However, the reality may be more complex. On the one hand, most Americans trust their personal physicians and the broader medical community to provide them with accurate information about COVID-19 (Funk, 2020; Sanger-Katz, 2020).

On the other hand, however, trust in scientific experts is far from unanimous (Gauchat, 2012; Motta, 2018), and those who distrust scientific experts have been shown to be particularly resistant to evidence-based messaging originating from expert sources (e.g., Merkley, 2020). Correspondingly, as White House Coronavirus Task Force Director Dr. Anthony Fauci recognized in a May 2020 interview, anti-science attitudes pose a potential threat to expert-sourced health communication about a vaccine for COVID-19 (Cohen 2020). Therefore, the CDC plans to -- in Fauci's words -- present Americans with pro-vaccine messages from "people they can relate to in the community."

Although we think it is important to account for differences in message source when testing the effectiveness of different provaccine arguments, we are somewhat agnostic as to whether or not expert (e.g., medical doctors presenting scientific facts) or lay (e.g., ordinary people recounting their experiences with the virus) sourced arguments will be more effective at encouraging vaccine uptake. With that in mind, we propose RQ1a-b. 
RQ1: Will expert (vs. lay) sourced pro-vaccine messages

(a) be more effective at increasing intention to vaccinate across message frames, and/or (b) when paired with specific message frames?

Additionally, we recognize that messages are likely to vary in the amount of information they provide about the safety and efficacy of clinical trials. Operation Warp Speed is promising to deliver a COVID-19 vaccine for public consumption in a record time frame (Cohen, 2020), and has raised public concerns about whether or not the final product (s) may be "rushed" to market (Hastline, 2020). As a result, communicators may try to preempt this concern via inoculation (McGuire, 1961), also referred to as "pre-bunking."

In a pre-bunking communication paradigm, communicators recognize the potential for the public to be misinformed about (in this case) the rigors of clinical trials, and attempt to provide them with information to the contrary. Pre-bunking has been shown to be effective at reducing misinformation about climate change (Cook et al.,2017; Van der Linden et al., 2017) and public health (see: Compton et al., 2016 for an extensive review), and has been suggested as a potential communication mechanism for combating misinformation about the novel coronavirus (Habersaat et al., 2020; Van Bavel et al., 2020).

However, pre-bunking necessarily implies providing the public with the very misinformation it hopes to counteract (similar to inoculation on a biological level). It therefore risks potentially "backfiring;" e.g., that people accept the misinformation presented; see (Caulfield, 2020). Additionally, it is unclear whether or not pre-bunking efforts are successful at moving opinion about the novel coronavirus; for which concerns about the rigors of clinical trials, given widespread public attentiveness to the virus (Jurkowitz and Mitchell, 2020), may already be highly salient. Further, some studies suggest that fact-based (as opposed to logic-based) pre-bunking efforts may fail to move opinion (e.g., Vraga et al., 2020).

Consequently, it is unclear whether or not messages that attempt to preempt concerns about the speed of vaccine development will be effective at increasing vaccine intentions. It is also unclear whether or not pro-vaccine messages paired with that information might be comparatively more effective than messages not including that information. With this in mind, we propose RQ2:

RQ2: Will pre-bunking efforts (a) be more effective at increasing intention to vaccinate across frames, and/or (b) when paired with specific message frames?

\section{Could Partisan Polarization in Vaccination Intentions Moderate Treatment Effects?}

Finally, as some recent perspectives on the 2020 US general election have noted (e.g., Motta, 2020; Tyson et al., 2020), COVID-19 vaccination intentions have become highly politicized (see also: Callaghan et al., n.d.). Whereas about three quarters of Democrats and Republicans alike planned to receive a vaccine at the pandemic's early stages (April 2020), Republicans' intentions to vaccinate dropped off substantially from June through September (Motta, 2020; see also O’Keefe, 2020).

This trend appeared to reverse however, by early Fall, as partisan elites came to take clearer positions on vaccinerelated issues. Following an uptick in elite polarization in vaccine-related rhetoric-e.g., President Trump's (the Republican candidate) frequent promise to deliver a vaccine by Election Day; Senator Harris' (the Democratic candidate's running mate) apprehension to receive a vaccine approved by the Trump administration, as noted in the cycle's Vice Presidential Debate; and concerns that the Trump administration might pressure government agencies to pursue emergency use authorization for a vaccine prior to the conclusion of clinical trials-Republicans' vaccination rebounded, while Democrats' intentions fell sharply, in October 2020 (Motta, 2020).

Consequently, we might ask whether or not the treatment effects we expect to observe (see above) might vary by political partisanship. As our study was conducted in mid June 2020 (more on this shortly), we might expect Republicans to be less receptive than Democrats to our messages. This possibility could have important and negative consequences for vaccine uptake, as it would imply that a substantial portion of the American population might miss our efforts to encourage vaccination. Consequently, we pose the following research question:

RQ 3: Will the efficacy of pro-vaccination messages that vary in source, frame, and/or the inclusion of prebunking information be more effective for Democrats, vs. Republicans?

\section{Experimental Design}

To test our theoretical expectations, we devised a large survey experiment varying three different aspects of COVID-19 vaccine promotion messages. Respondents were randomly assigned to read a short pro-vaccine opinion piece, varying 1 ) the frame (personal health risks, collective public health consequences of not vaccinating, and economic costs), 2) the message source (i.e., lay vs. expert sources), and 3) the presence or absence of pre-bunking information highlighting the rigors of clinical trials prior to reading the opinion piece. A final group of respondents, serving as our control group, were randomly assigned to receive none of these messages, and instead read a short story about baseball.

All respondents not assigned to the control group were told that we would like them to "read a short newspaper opinion piece about the importance of receiving a COVID-19 vaccine, when it becomes available." Respondents assigned to receive pre-bunking information about the rigors of clinical trials were also told that "even though the vaccine is likely to be developed in an accelerated time frame," that it will nevertheless undergo rigorous clinical testing. In those conditions, we told respondents that "this means that scientists will observe whether or not people experience side effects from the vaccine right away, as well as over the course of several months."

Table 1 summarizes the key elements of our experimental design, including the number of respondents assigned to each of the twelve conditions ( $3 \times 2 \times 2$ design), and the title of each opinion piece. Full text for each experimental treatment 
TABLE 1 | Experimental design summary

\begin{tabular}{|c|c|c|}
\hline Frame & Source (lay) & Source (expert) \\
\hline $\begin{array}{l}\text { Personal health risk } \\
\qquad \begin{array}{l}\text { No clinical trial info } \\
N=504 \text { (lay) } \\
N=522 \text { (expert) }\end{array}\end{array}$ & $\begin{array}{l}\text { Thinking about skipping the COVID-19 vaccine? Take it from } \\
\text { someone who had the virus: That's a bad idea }\end{array}$ & $\begin{array}{l}\text { Thinking about skipping the COVID-19 vaccine? You're putting your } \\
\text { health at risk }\end{array}$ \\
\hline $\begin{array}{l}\text { Clinical trial info } \\
\text { (pre-bunk) } \\
\begin{aligned} \mathrm{N}=497 \text { (lay) } \\
\mathrm{N}=546 \text { (expert) }\end{aligned}\end{array}$ & $\begin{array}{l}\text { Corey Miller is an accountant from Austin, TX, who suffered } \\
\text { complications from the novel coronavirus in March } 2020\end{array}$ & Dr. Corey Miller is a Medical Doctor at the University of Texas - Austin \\
\hline $\begin{array}{l}\text { Economic costs } \\
\qquad \begin{aligned} \text { No clinical trial info } \\
N=505 \text { (lay) } \\
N=485 \text { (expert) }\end{aligned}\end{array}$ & $\begin{array}{l}\text { Thinking about skipping the COVID-19 vaccine? Take it from } \\
\text { someone who lost their job: That's a bad idea }\end{array}$ & $\begin{array}{l}\text { Thinking about skipping the COVID-19 vaccine? Prepare for a slower } \\
\text { economic recovery }\end{array}$ \\
\hline $\begin{array}{l}\text { Clinical trial info } \\
\text { (pre-bunk) } \\
\begin{aligned} \mathrm{N}=510 \text { (lay) } \\
\mathrm{N}=471 \text { (expert) }\end{aligned}\end{array}$ & $\begin{array}{l}\text { Corey Miller is an accountant from Austin, TX, who suffered job } \\
\text { loss as a result of the novel coronavirus in March } 2020\end{array}$ & $\begin{array}{l}\text { Dr. Corey Miller is a Professor in the Department of Economics at the } \\
\text { University of Texas-Austin }\end{array}$ \\
\hline $\begin{array}{l}\text { Collective health } \\
\text { consequences } \\
\begin{array}{l}\text { No clinical trial info } \\
N=496 \text { (lay) } \\
N=506 \text { (expert) }\end{array}\end{array}$ & $\begin{array}{l}\text { Thinking about skipping the COVID-19 vaccine? Tell that to people } \\
\text { who depend on you to get vaccinated }\end{array}$ & $\begin{array}{l}\text { Thinking about skipping the COVID-19 vaccine? Prepare for more } \\
\text { deaths and hospitalizations }\end{array}$ \\
\hline $\begin{array}{l}\text { Clinical trial info } \\
\text { (pre-bunk) } \\
\begin{aligned} N=493 \text { (lay) } \\
N=533 \text { (expert) }\end{aligned}\end{array}$ & $\begin{array}{l}\text { Corey Miller is an accountant from Austin, TX, who is currently } \\
\text { undergoing chemotherapy treatments for lung cancer }\end{array}$ & $\begin{array}{l}\text { Dr. Corey Miller is an Austin, TX based Pharmaceutical Consultant for } \\
\text { Johnson and Johnson, a United States. company developing a vaccine } \\
\text { for COVID-19 }\end{array}$ \\
\hline
\end{tabular}

condition can be found in the Supplementary Material. Note also that we gave all fictitious sources the same name, one that we selected as to not clearly identify the communicator's gender. We also attributed all sources to be from the same geographic location (Austin, Texas) in order to standardize perceptions of "on the ground" COVID prevalence in each area; e.g., because communicators discussing personal risks may be more/less likely to be dismissed, based on whether or not they live in a place where COVID is comparatively more prevalent. As a "blue" (politically liberal) city in a "red" (politically conservative) state, Austin also helps us avoid the possibility of source/message discrediting on the basis that the disease' prevalence and severity is a "blue" or "red" area problem -- a point of popular contention at the time we fielded this study (e.g., Bump, 2020).

In addition to the design elements summarized in Table 1, we clarify that this is an intent to treat experimental design. We required that all respondents-irrespective of experimental condition, and including those assigned to the control condition-spend at least 15 seconds on the page containing our short opinion piece (or the baseball story, in the case of the control).

Finally, balance tests included in the Supplementary Material (Supplementary Figure S1) reveal that our randomization protocol produced treatment groups that were highly similar in both demographic composition (e.g., age, race, gender, educational attainment), and their prior attitudes toward COVID vaccine safety. We find statistically significant evidence of imbalance in just two out of twelve conditions (both of the personal health risk appeals originating from medical experts), and on just one demographic dimension (gender). Consequently, (Supplementary Table S3) in the Supplementary Material, we replicate all analyses presented in the main text conditioning on respondents' gender, and uncover an identical pattern of effects.

\section{Data and Measures}

Data for this study come from a demographically representative survey sample of $\mathrm{N}=7,064$ United States adults, recruited between July 8-20, 2020. Respondents were invited to participate in our survey via Lucid Theorem's large online opt-in panel via quota sampling, in order to ensure demographic representativeness on respondents' age, race, gender, educational attainment, income, and geographic region. Lucid initially invited $\mathrm{N}=10,020$ individuals to participate in this study, yielding a completion rate of $70 \%$. Further, Lucid data has been found replicate demographic and experimental findings, and that Lucid samples are more nationally representative than traditional convenience samples on various demographic, political, and psychological factors (Coppock and McClellan, 2019). Researchers in public health and health politics have previously published papers using Lucid data (e.g., Callaghan et al., 2019; Lunz Trujillo et al., 2020).

The key outcome variable in our analyses is an indicator of whether or not respondents intend to vaccine against COVID-19, once a vaccine becomes available. Respondents were asked "When a vaccine for the novel coronavirus (COVID-19) becomes widely available, how likely are you to request to be vaccinated?" Respondents then indicated whether or not they are "very likely," "somewhat likely," "not too likely," or "not likely at all" to vaccinate. We score this variable to range from 1-4, such that a score of 4 indicates being "very likely" to vaccinate.

We note that just $42 \%$ of respondents indicated that they were "very likely" to get vaccinated, while an additional $30 \%$ indicated 


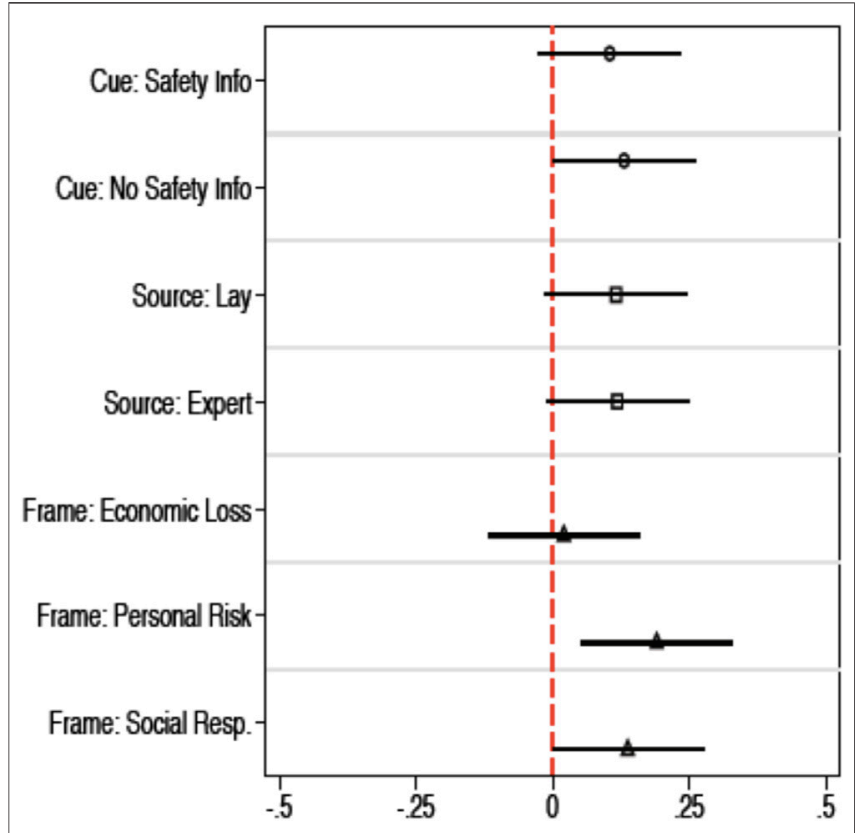

FIGURE 1 | Independent effects of frame, source and clinical Info. Cue (pooled). Note. Ordered logistic regression coefficients presented, with 95\% confidence intervals. Outcome variable is an ordinal indicator of COVID-19 vaccine intention (with higher scores reflecting increased intention to vaccinate). Coefficients which do not intersect with the dashed red line are statistically significant from control group effects at the $p<0.05$, level (two-tailed). Results are derived from three models (denoted by different shapes, in the figure) which regressing vaccine intention on indicators denoting assignment to each design element listed on the lefthand side of the figure; pooled across all other design elements.

that they are somewhat likely to do so. Note also that we include the term "request" in this question, as vaccinating against COVID-19 entails, for most adults, actively choosing to vaccinate, during a period designated by state and local governments, and making appropriate logistical preparations therein.

The primary independent variables in our analyses are binary indicators of the experimental conditions to which respondents were randomly assigned (see: Table 1). To study the effects of each design element in isolation, we at times pool (or "collapse") these indicators across all other design elements. For example, combined indicators denoting assignment to each of the three frames we varied pool across both sources and the presence/ absence of a pre-bunking text. To study the effects of each design element in context, we create twelve dichotomous indicators of assignment to each of the cells listed in Table 1, with the control serving as a reference group in all analyses.

Finally, to assess whether or not our messaging strategies are effective at encouraging vaccination among those most skeptical of a potential COVID vaccine's safety and efficacy, we asked respondents: "When a vaccine for the novel coronavirus (COVID-19) becomes widely available, do you think that its potential benefits will outweigh potential risks, that its potential risks might outweigh potential benefits, or haven't you thought much about it?" This question was administered prior to our experimental treatments. For analytical simplicity, and to preserve a sufficiently large sample size in each experimental treatment condition, we dichotomize respondents as being "COVID vaccine skeptics" if they either indicate that the vaccine's risks will outweigh its benefits, or if they haven't thought much about the issue. Scored this way, 54\% of our sample are skeptical of the vaccine's safety.

\section{RESULTS}

\section{Pooled Framing Effects}

We begin our analysis by offering an assessment of whether or not each framing tactic-independently of each other design element alteration-was effective at increasing the general public's intentions to get vaccinated against COVID-19. In this initial and "fully aggregated" approach, we isolate the effects of each framing alteration by pooling all other varied elements together.

Figure 1 displays the results of an ordered logistic regression model regressing the four-point vaccine intention variable on an indicator of experimental framing condition assignment (rows 1-3), pooled across source and clinical trial information manipulations. These serve as an initial test of Hypotheses $1-3$. Hollowed circles correspond to the unique effects of each frame, with 95\% confidence intervals extending from each one. Estimates which fall to the right of the dashed line, and whose confidence intervals do not intersect with it, suggest a statistically significant increase in vaccine intention.

We replicate this approach to isolate the unique effects of message source (rows 5-6) and whether or not the message included information about the rigors of clinical trials (rows 7-8). These serve as initial tests of RQ1a and RQ2a. The presence of significant effects in these more-exploratory analyses could point us in the direction of potential message effectiveness moderators to consider when disaggregating design elements, later on. We note that all $p$ values presented in the pages that follow are two-tailed.

Figure 1 demonstrates that both the personal health risk frame $(\mathrm{B}=0.19, p<0.01)$ and collective public health consequences frame $(B=0.14, p=0.05)$ are positively and significantly associated with increased intention to vaccinate. This provides strong evidence in favor of $\mathrm{H} 1$ and $\mathrm{H} 2$.

Of course, ordered logistic regression coefficients provide little sense of the substantive magnitude of these effects. Consequently, we present predicted probabilities that reflect the increase in indicating that respondents are "very likely" to vaccinate in each of these treatments (vs. the control). We focus on the "very likely" ordinal category, as we expect these individuals to be the most likely to intend to vaccinate, once a vaccine becomes available.

Substantively, assignment to the personal health risk frame (pooled across conditions) is associated with a $5 \%$ increase in the likelihood that respondents are "very likely" to pursue the vaccine; from $39 \%$ in the control group, to $44 \%$ to those assigned to read messages with this frame. Likewise, assignment to the collective health consequences condition is associated with a $4 \%$ increase in indicating that respondents are "very likely" to receive the vaccine, from $39 \%$ in the control to $43 \%$ in this frame.

Surprisingly, and contrary to our expectations, we find no evidence that economic frames are effective at moving vaccine intention. While the economic frame did nudge respondents in a 


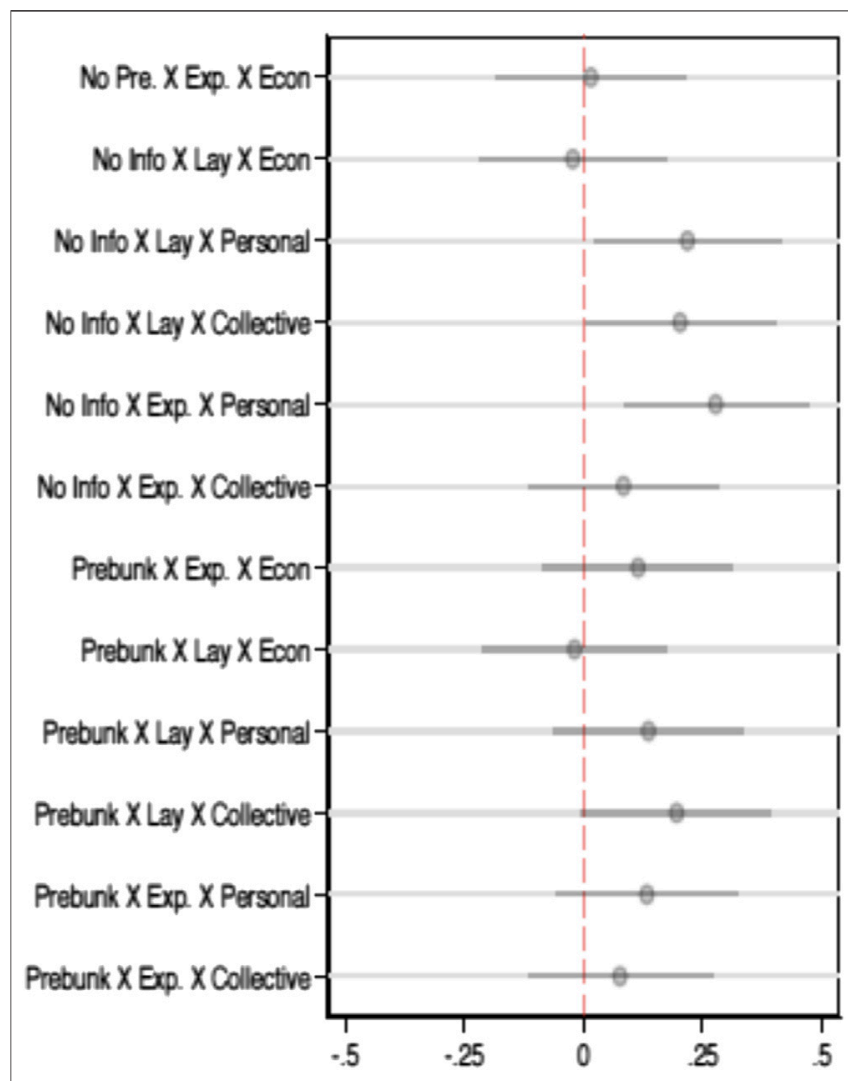

FIGURE 2 | Fully Disaggregated treatment Effects. Note. Ordered logistic regression coefficients presented, with 95\% confidence intervals. Outcome variable is an ordinal indicator of COVID-19 vaccine intention (with higher scores reflecting increased intention to vaccinate). Coefficients which do not intersect with the dashed red line are statistically significant from control group effects at the $p<0.05$, level (two-tailed). Results are derived from a model regressing vaccine intention on indicators denoting assignment to each source, by frame, by clinical information manipulation listed on the lefthand side of the figure.

pro-vaccination direction $(B=0.02)$, the effect was not statistically distinguishable from zero $(p=n . s$.$) .$

Concerning our research questions, Figure 1 presents no evidence in favor of the idea that sources originating from expert (vs. lay) sources -- pooled across all other design elements -- vary in their effectiveness (RQ1a). Vaccination intention was not significantly different from that of the control group for respondents assigned to read messages from either lay or expert sources (in both cases, $\mathrm{B}=0.12, p=n . s$.).

Moreover, while we find that pre-bunking information (RQ2a) is positively associated with increased vaccination intention $(\mathrm{B}=0.10)$, the effects were not statistically significant $(p=n . s$.). In fact, we find some evidence in favor of the idea that not including pre-bunking information is associated with increased vaccination intention, versus the control group ( $\mathrm{B}=0.13, p<0.05$ ). However, we caution that these differences do not necessarily rule out the potential effectiveness of pre-bunking; given the overlapping confidence intervals presented in Figure 1.

\section{Disaggregated Framing Effects}

Next, we consider the possibility that the framing effects observed in Figure 1 may be more efficacious when originating from expert or lay sources (RQ1b), and/or when featuring (or not featuring) pre-bunking information about the rigors of clinical trials (RQ2b). Figure 2 presents the results of an ordered logistic regression model regressing vaccine intentions on our fully disaggregated treatment indicators (see: Table 1), and is otherwise analogous in interpretation to Figure 1.

The results suggest that, among conditions that featured no prebunking information, appeals to the personal health risks of not vaccinating were positively and significantly associated with increased vaccine intention for both expert $(B=0.28, p<0.01)$ and lay $(B=0.22, p=0.03)$ sources. Both of these effects were substantively similar in size, leading to a $7 \%$ and $6 \%$ increase in the likelihood of being "very likely" to receive the vaccine, respectively.

Additionally, among the conditions featuring no pre-bunking manipulation, we find that while both expert $(B=0.08)$ and lay $(B$ $=0.20$ ) sourced messages about the collective health risks of failing to vaccine are associated with increased vaccine intentions, the effects are only statistically significant for the latter $(p=0.05)$. Exposure to lay messages about the collective health risks of not vaccinating are associated with a $5 \%$ increase in the likelihood of being "very likely" to vaccinate.

Among conditions that do feature pre-bunking information, we uncover only one statistically significant increase in vaccine intention across variations on the source and arguments presented in each message. In this case, only messages emphasizing the collective health risks of failing to vaccinate, sourced by ordinary people, produced statistically significant increases in vaccine intention $(\mathrm{B}=0.19, p=0.05)$. This is perhaps unsurprising, given the slight decrease in message effectiveness we noted when addressing RQ2a when presenting pooled experimental effects in Figure 1. Further, as we might also expect based on the pattern of results presented in Figure 1, we uncover no statistically significant effects of messages including frames concerning the economic costs of not vaccinating.

Taken together, the disaggregated results suggest that the effectiveness of messages emphasizing personal health risk-and, to a lesser degree, collective health risks-are consistent across message sources. However, the addition of information designed to assuage respondents' potential fears about the safety of expedited clinical trials appear to have weakened treatment effects across the board. Of course, we hesitate to discredit messaging strategies on the basis of post hoc conclusions about differential source or clinical trial information, and recognize that our messaging strategy is just one of many that health communicators might employ.

Of course, we caveat that the effects observed in Figures 1, 2 increase the likelihood that people opt to vaccinate by just a handful of percentage points. While we recognize that these effects are small in substantive magnitude, we also recognize that-as noted earlier-the rate at which Americans intend to vaccinate aginst COVID-19 is precariously close to the compliance threshold necessary to achieve population immunity. Consequently, we believe that even effects that are small in substantive magnitude could play an instrumental role in putting the spread of COVID-19 into decline. 
On balance, then, across the results presented in Figures 1, 2, we conclude that-based on how we formulated each design element in the present study-messages emphasizing the personal and collective health risks of not vaccinating are particularly efficacious at increasing vaccine intention; especially if those messages originate from non-expert sources, and avoid discussing attempting to preempt concerns about the rigors of clinical trials.

\section{Moderation by Political Partisanship?}

Additionally, given documentation of COVID-19 vaccine politicization, we ask (RQ3) whether or not the effects documented thus far might be substantively and/or statistically stronger for self-identified Democrats (vs. Republicans). To test this, we interacted each treatment indicator presented in Figures 1, $\mathbf{2}$ with a standard seven-point measure of partisan identification (ranging from $1=$ Strong Democrat to $7=$ Strong Republican).

In analyses (Supplementary Table S4) presented in the Supplementary Material, we find no evidence that our treatment effects were moderated by political partisanship. Across both the pooled (see: Figure 1) or fully disaggregated models (see: Figure 2), all interactions fail to even approach statistical significance at the $p=0.10$ level, two-tailed. Consequently, while it is certainly the case that vaccine intentions were a politically polarizing issue at the time we conducted our study, we find no evidence that these differences influenced treatment effectiveness.

\section{Subgroup Analyses for COVID Vaccine Skeptics}

Finally, we conclude our analysis by considering whether or not any of the experimental manipulations presented thus far might be effective at winning over individuals who are skeptical that a vaccine for COVID-19 will be safe and effective. As this group may be particularly likely to refuse vaccination, we think that is important to consider whether or not some messages may be more effective than others at winning over this group.

Supplementary Tables $\mathbf{S 2}$ replicates the models used to build Figures 1, 2 (respectively), limiting analysis to just individuals who express hesitation about the relative benefits of a COVID19 vaccine, relative to its potential risks. Note that we do not interact our binary indicator of COVID vaccine skepticism with assignment to each experimental condition, as we are less interested in whether or not treatments are comparatively more or less effective for this group; we would expect skeptics, across the board, to be less receptive to persuasive messaging attempts of all varieties than those who are less skeptical. Instead, we aim to determine whether or not any of these messaging strategies are effective at all at increasing vaccination intentions for this group.

Both the pooled and disaggregated (Supplementary Tables S1) results suggest that our treatments had a limited influence on vaccination intentions for vaccine skeptics. We find that just one treatment-messages originating from lay sources, emphasizing the collective health risks of failing to vaccinate, and that feature pre-bunking information about the rigors of expedited clinical trials-was significantly associated with increased vaccination intention ( $\mathrm{B}=0.27, p=0.04)$.

These results suggest that, while personal and collective health risk frames are generally effective at convincing Americans to consider vaccinating against COVID-19, these effects are likely limited to those who do not question the vaccine's potential safety and efficacy. As a result, efforts to bolster public trust in the vaccine, once it becomes available, could prove critical in influencing public receptiveness to the types of messages we study in this research. Consistent with this view, we note that the lone manipulation successful at moving vaccination intention for skeptics is one that provided information about the safety and efficacy of expedited clinical trials.

\section{CONCLUSION}

In this study, we find that messages emphasizing the personal risks of failing to vaccinate against COVID, as well as the potential collective public health consequences of low vaccine uptake, are effective at convincing Americans to plan to get a COVID vaccine, once one becomes available. These effects are similar for Democrats, Republicans, and Independents alike. Consequently, we recommend that health communicators aiming to boost COVID vaccine uptake consider employing one or both of these frames as part of their strategic communication efforts.

Exploratory analyses reveal that both lay and expert sources can communicate these messages effectively, although we find some evidence in favor of the idea that "ordinary people" may be comparatively more effective. In addition, we note that attempts to preempt (via pre-bunking) concerns about the rigors of clinical trials offer no discernible benefit above not providing this information, and find preliminary evidence suggesting that pre-bunking may limit the effectiveness of personal and public health frames. Finally, we find little evidence that frames highlighting the economic costs of failing to vaccine are effective at increasing vaccine intention. This is somewhat surprising, given the pandemic's far-reaching economic consequences, and the effectiveness of similar appeals documented in previous research.

Of course, we want to be cautious and note some important limitations of this study, and our recommendations for future health communication efforts. First, our study is necessarily limited in not only the message design elements it alters (framing, source, and pre-bunking appeals), but also in the design of each of those elements themselves. While we are confident that the personal and collective health risk frames presented in this piece are indeed effective, we of course cannot rule out the possibility that 1 ) other variations of these frames are equally or more effective, 2) that messages similar to ours communicated by different expert vs. lay sources might differ from the disaggregated results presented in Figure 2 (e.g., by varying expert/lay sources within frames), and/or 3) that other frames not considered in this piece might also be effective at moving intention.

Relatedly, we cannot, in this piece, offer up a particular communication approach as being comparatively more effective 
than others that we find to be effective in boosting vaccination intentions. As Figures 1, 2 demonstrate, several communication efforts boost vaccination intentions more than we would expect by random chance. However, none of the efforts we deem effective at boosting vaccination intentions are statistically distinguishable from one another. Consequently, although effect sizes may differ in magnitude, we consider all statistically significant treatment effects to have a similar substantive effect on vaccination intentions, relative to taking no action at all.

We also caution that the language that expert vs. non-expert sources use in these messages necessarily varies across treatments. Consequently, we cannot definitively rule out the possibility that effects related to message source that we observe may be influenced in part by rhetorical style. In particular, our study cannot guarantee that effects attributed to messages sourced by ordinary people may be, at least in part, the result of those messages being more personal in their substantive focus. While our treatments avoid the use of medical and statistical jargon in the experimental conditions (please see the Supplementary Material for full treatment text), we nevertheless urge caution when interpreting these results; especially those related to message source.

Despite these limitations, we are able to make recommendations about what does effectively increase vaccine intention. However, we are hesitant to rule out the possibility that what does not appear to do so in our study might be more effective under other conditions. We see our research as not the final word on how to effectively boost COVID vaccine uptake, but instead as a starting point for future efforts to unpack the conditional effectiveness of personal and collective public health risk frames, and to consider altering other design elements not presented in this paper. Conjoint experiments may be a particularly useful route for testing these possibilities in future research by varying exposure to dozens of different design elements -- paired with alterations within each design element -- to a sufficiently large sample.

Given the importance of widespread vaccination against COVID-19, several additional directions for future research are particularly important to pursue. First, while our analysis suggests that messages from ordinary Americans and experts can both be effective (with slightly better outcomes for ordinary individuals), more work is needed to determine if certain types of communicators within each category are better at conveying messages that others. On the non-expert side, understanding the value of contextual cues of the messenger (e.g., gender, partisanship, and race) will be critical to identifying the best communicators for the importance of vaccination. So too might the role of celebrity endorsements for pro-vaccine messages; a tactic the CDC has considered pursuing. On the expert side, it will be similarly important to understand for example if a message from Dr. Anthony Fauci is perceived as more or less effective than a message from the CDC, Dr. Deborah Birx, or any of the other experts involved in COVID-19 health communication.

Beyond message sources, additional research is also needed to determine how repeated message exposure and exposure to countervailing messages influence vaccination intention. While our experimental design is useful for isolating the effectiveness of specific pro-vaccine messages, we cannot capture the complexities of an information environment in which people will be presented with competing pro and anti-vaccination messages. Future research would benefit from exploring how COVID-19 vaccination messages interact and influence behavior.

Finally, it is critical to acknowledge that our experimental design did little to alter the planned behavior of individuals who were skeptical of the vaccine's safety and efficacy. Improving vaccination among those most hesitant could prove vital to reaching the immunization rates needed to achieve herd immunity. Future research should explore additional messaging strategies to improve vaccination intention in this group. Prior research by Lunz Trujillo et al. (2020) suggests that it could be particularly valuable to understand why skeptics are hesitant to vaccinate and to design targeted interventions to appeal specifically to these harder to move individuals. Given the safety and efficacy concerns of individuals in this hesitant group, particular emphasis could be placed on interventions designed to ease safety and efficacy concerns.

Even with these limitations and directions for future research however, our study provides a critical step forward in our understanding of how to improve COVID-19 vaccination compliance. Our research suggests that both lay and expert health communicators can improve vaccination rates by highlighting the personal health risks and collective public health consequences of COVID-19 vaccination, while suggesting that economic arguments may be less effective. Consequently, we hope to help to inform strategic health communication efforts to encourage the widespread vaccination necessary to stop the spread of COVID-19 and end the global pandemic.

\section{DATA AVAILABILITY STATEMENT}

The datasets generated for this study can be found here: https:// osf.io/2t9zb/

\section{ETHICS STATEMENT}

The studies involving human participants were reviewed and approved by Utah Valley University. The patients/participants provided their written informed consent to participate in this study.

\section{AUTHOR CONTRIBUTIONS}

MM, SS, TC, and KL-T were Responsible for project conceptualization, research design, and manuscript writeup. MM was also responsible for data cleaning, analysis, and preparation of the supplementary materials.

\section{SUPPLEMENTARY MATERIAL}

The Supplementary Material for this article can be found online at: https://www.frontiersin.org/articles/10.3389/fpos.2021.630133/ full\#supplementary-material. 


\section{REFERENCES}

Adams, J. G., and Walls, R. M. (2020). Supporting the health care workforce during the COVID-19 global epidemic. Jama 323 (15), 1439-1440. doi:10.1001/jama. 2020.3972

Arndt, J., and Goldenberg, J. L. (2017). Where health and death intersect: insights from a terror management health model. Curr. Dir. Psychol. Sci. 26 (2), 126-131. doi:10.1177/0963721416689563

Bartsch, S. M., O’Shea, K. J., Ferguson, M. C., Bottazzi, M. E., Wedlock, P. T., Strych, U., et al. (2020). Vaccine efficacy needed for a COVID-19 coronavirus vaccine to prevent or stop an epidemic as the sole intervention. Am. J. Prev. Med. 59 (4), 493-503.

Bean, M. (2020). COVID-19 is 3rd leading cause of death, STAT analysis suggests, Chicago, IL: Becker's Hospital Review.

Becker, E. (1973). The denial of death. New York, NY: Free Press.

Betsch, C., Böhm, R., and Chapman, G. B. (2015). Using behavioral insights to increase vaccination policy effectiveness. Policy Insights. Behav. Brain. Sci. 2 (1), 61-73. doi: $10.1177 / 2372732215600716$

Böhm, R., Betsch, C., and Korn, L. (2016). Selfish-rational non-vaccination: experimental evidence from an interactive vaccination game. J. Econ. Behav. Organ. 131, 183-195. doi:10.1016/j.jebo.2015.11.008

Britton, T. T., Ball, F., and Trapman, P. (2020). A mathematical model reveals the influence of population heterogeneity on herd immunity to SARS-CoV-2. Science 369 (6505), 846-849. doi:10.1126/science.abc6810

Bump, P. (2020). The shift of the coronavirus to primarily red states is complete-but it's not that simple. Available at: https://www.washingtonpost. com/politics/2020/06/24/shift-coronavirus-primarily-red-states-is-complete-itsnot-that-simple/ (Accessed June 24,2020 ).

Callaghan, T., A. Moghtaderi, J. A. Lueck, P. Hotez, U. Strych, A. Dor, et al. (2020). Correlates and disparities of intention to vaccinate against COVID-19. Soc. Sci. Med. 113638 .

Callaghan, T., Motta, M., Sylvester, S., Trujillo, K. L., and Blackburn, C. C. (2019). Parent psychology and the decision to delay childhood vaccination. Soc. Sci. Med. 238, 112407. doi:10.1016/j.socscimed.2019.112407

Cascella, M., Rajnik, M., Cuomo, A., Dulebohn, S. C., and Di Napoli, R. (2020). Features, Evaluation and Treatment Coronavirus (COVID-19), Treasure Island, FL: StatPearls Publishing.

Caulfield, T. (2020). Does debunking work? Correcting COVID-19 misinformation on social media. OSF [Epub ahead of print]. doi:10.31219/osf.io/5uy2f

CDC Centers for Disease Control and Prevention (2020). Coronavirus Disease 2019 (COVID-19). Available at: https://www.cdc.gov/coronavirus/2019-ncov/ prevent-getting-sick/prevention.html (Accessed August 26, 2020).

Cohen, E. (2020). Fauci says Covid-19 vaccine may not get US to herd immunity if too many people refuse to get it. Available at: https://www.cnn.com/2020/06/ 28/health/fauci-coronavirus-vaccine-contact-tracing-aspen/index.html (Accessed June 29, 2020).

Compton, J., Jackson, B., and Dimmock, J. A. (2016). Persuading others to avoid persuasion: inoculation theory and resistant health attitudes. Front. Psychol. 7, 122. doi:10.3389/fpsyg.2016.00122

Cook, J., Lewandowsky, S., and Ecker, U. K. (2017). Neutralizing misinformation through inoculation: exposing misleading argumentation techniques reduces their influence. PloS One 12 (5), e0175799. doi:10.1371/journal. pone.0175799

Coppock, A., and McClellan, O. A. (2019). Validating the demographic, political, psychological, and experimental results obtained from a new source of online survey respondents. Res. Politics 6 (1), 2053168018822174. doi:10.1177/ 2053168018822174

Cornwall, W. (2020). Just 50\% of Americans plan to get a COVID-19 vaccine. Here's how to win over the rest. Science doi:10.1126/science.abd6018

COVID-19 Dashboard (2020). Center for Systems Science and Engineering, Baltimore, MD: Johns Hopkins University.

Funk, C. (2020). Polling shows signs of public trust in institutions amid the pandemic. Available at: https://www.pewresearch.org/science/2020/04/07/ polling-shows-signs-of-public-trust-in-institutions-amid-pandemic/ (Accessed April 26, 2020).
Gauchat, G. (2012). Politicization of science in the public sphere: a study of public trust in the United States, 1974 to 2010. Am. Socio. Rev. 77 (2), 167-187. doi:10. $1177 / 0003122412438225$

Greenberg, J., Pyszczynski, T., and Solomon, S. (1986). "The causes and consequences of a need for self-esteem: a terror management theory," in Public self and private self. Editor R. Baumeister (New York, NY: Springer), 189-212. doi:10.1007/978-1-4613-9564-5_10

Habersaat, K. B., Betsch, C., Danchin, M., Sunstein, C. R., Böhm, R., Falk, A., and Fischer, E. F. (2020). Ten considerations for effectively managing the COVID19 transition. Nat. Hum. Behav. 4, 677-687. doi:10.1038/s41562-020-0906-x

Haglin, K., Chapman, D., Motta, M., and Kahan, D. (2019). How localized outbreaks and changes in media coverage affect zika attitudes in national and local contexts. Health Commun., 35 (13), 1686-1697. doi:10.1080/ 10410236.2019.1662556

Hastline, W. (2020). The risks of rushing a COVID-19 vaccine. Available at: https://www.scientificamerican.com/article/the-risks-of-rushing-a-covid-19vaccine/ (Accessed June 22, 2020)

Havers, F. P., Reed, C., Lim, T., Montgomery, J. M., Klena, J. D., Hall, A. J., et al. (2020). Seroprevalence of antibodies to SARS-CoV-2 in 10 sites in the United States, March 23-May 12, 2020. JAMA Intern. Med, 180 (12), 1576-1586.

Jurkowitz, M., and Mitchell, A. (2020). Older Americans continue to follow COVID-19 news more closely than younger adults. Available at: https:// www.journalism.org/2020/04/22/older-americans-continue-to-follow-covid19-news-more-closely-than-younger-adults/ (Accessed April 22, 2020).

Kahan, D. (2010). Fixing the communications failure. Nature 463 (7279), 296-297. doi:10.1038/463296a

Kahneman, D. (2011). Thinking, fast and slow, City of Westminster, United Kingdom: Penguin Books Limited.

Kane, R. L., Johnson, P. E., Town, R. J., and Butler, M. (2004). A structured review of the effect of economic incentives on consumers' preventive behavior. Am. J. Prev. Med. 27 (4), 327-352. doi:10.1016/j.amepre.2004.07.002

Kochar, R. (2020). Unemployment rose higher in three months of COVID-19 than it did in two years of the great recession. Available at: https://www.pewresearch. org/fact-tank/2020/06/11/unemployment-rose-higher-in-three-months-of-covid19-than-it-did-in-two-years-of-the-great-recession/ (Accessed June 11, 2020).

Kwok, K. O., Lai, F., Wei, W. I., Wong, S. Y. S., and Tang, J. W. (2020). Herd immunity-estimating the level required to halt the COVID-19 epidemics in affected countries. J. Infect. 80 (6), e32-e33. doi:10.1016/j.jinf.2020.03.027

Li, M., Taylor, E. G., Atkins, K. E., Chapman, G. B., and Galvani, A. P. (2016). Stimulating influenza vaccination via prosocial motives. PloS One 11 (7), e0159780. doi:10.1371/journal.pone.0159780

Long, B., Brady, W. J., Koyfman, A., and Gottlieb, M. (2020). Cardiovascular complications in COVID-19. Am. J. Emerg. Med. 38 (7), 1504-1507. doi:10. 1016/j.ajem.2020.04.048

Lunz Trujillo, K., Motta, M., Callaghan, T., and Sylvester, S. (2020). Correcting misperceptions about the MMR vaccine: using psychological risk factors to inform targeted communication strategies. Polit. Res. Q. 1, 15.

Lunz Trujillo, K., and Motta, M. (2020). Many vaccine skeptics plan to refuse a COVID-19 vaccine, study suggests, Washington, DC: US News \& World Report.

McGuire, W. J. (1961). Resistance to persuasion conferred by active and passive prior refutation of the same and alternative counterarguments. J. Abnorm. Soc. Psychol. 63 (2), 326. doi:10.1037/h0048344

Merkley, E. (2020). Anti-intellectualism, populism, and motivated resistance to expert consensus. Publ. Opin. Q. 84 (1), 24-48. doi:10.1093/poq/nfz053

Motta, M., and Goren, P. (2020). Apolitical values and compliance with government-recommended prosocial health behavior. OSF 1-15. [Epub ahead of print]. doi:10.31235/osf.io/hxjk5

Motta, M. (2020). President Trump promised a coronavirus vaccine by election day. That politicized vaccine intentions. US Elect. Analy. Econo. Time.

Motta, M. (2018). The dynamics and political implications of anti-intellectualism in the United States. Am. Polit. Res. 46 (3), 465-498. doi:10.1177/ $1532673 \times 17719507$

Newport, F. (2020). 10 key findings: public opinion on coronavirus. Gallup. 20 march 2020. Available at: https://news.gallup.com/opinion/polling-matters/ 296681/ten-keyfindings-public-opinion-coronavirus.aspx (Accessed March 20, 2020). 
Nyhan, B., and Reifler, J. (2015). Does correcting myths about the flu vaccine work? An experimental evaluation of the effects of corrective information. Vaccine 33 (3), 459-464. doi:10.1016/j.vaccine.2014.11.017

O'Keefe, S. M. (2020). One in three Americans would not get coronavirus vaccine. Gallup. Available at: https://news.gallup.com/poll/317018/one-threeamericans-not-covid-vaccine.aspx (Accessed August 7, 2020).

Reimann, N. (2020). A second wave of shutdowns has arrived-here's where businesses are closing up (again). Available at: https://www.forbes.com/sites/ nicholasreimann/2020/07/06/a-second-wave-of-shutdowns-has-arrived-hereswhere-businesses-are-closing-up-again/\#4b2fb1614602 (Accessed July 6, 2020).

Sanger-Katz, M. (2020). On coronavirus, Americans still trust the experts. The New York Times, A20, July, 27.

Taylor, S., Landry, C., Paluszek, M., Fergus, T. A., McKay, D., and Asmundson, G. J. (2020). Development and initial validation of the COVID stress scales. J. Anxiety Disord. 72, 102232. doi:10.1016/j.janxdis.2020.102232

Thigpen, C. L., and Funk, C. (2020). Most Americans expect a COVID-19 vaccine within a year; $72 \%$ say they would get vaccinated. Available at: https://www. pewresearch.org/fact-tank/2020/05/21/most-americans-expect-a-covid-19vaccine-within-a-year-72-say-they-would-get-vaccinated/ (Accessed May 21, 2020).

Tyson, C., Johnson, C., and Funk, C. (2020). US public now divided on whether or not to get coronavirus vaccine. Washington, DC: Pew Research Center.

Van Bavel, J. J., Baicker, K., Boggio, P. S., Capraro, V., Cichocka, A., Cikara, M., and Drury, J. (2020). Using social and behavioural science to support COVID-19 pandemic response. Nat. Hum. Behav. 4 (5), 460-471. doi:10.1038/s41562-0200884-z

Van der Linden, C., and Savoie, J. (2020). Does collective interest or self-interest motivate mask usage as a preventive measure against COVID-19?. Can J Polit. Sci., 1-7. doi:10.1017/S0008423920000475
Van der Linden, S. L., Clarke, C. E., and Maibach, E. W. (2015). Highlighting consensus among medical scientists increases public support for vaccines: evidence from a randomized experiment. BMC Pub. Health 15 (1), 1-5. doi:10.1186/s12889-015-2541-4

Van der Linden, S., Leiserowitz, A., Rosenthal, S., and Maibach, E. (2017). Inoculating the public against misinformation about climate change. Glob Chall 1 (2), 1600008. doi:10.1002/gch2.201600008

Vraga, E. K., and Bode, L. (2017). Using expert sources to correct health misinformation in social media. Sci. Commun. 39 (5), 621-645. doi:10.1177/ 1075547017731776

Vraga, E. K., Kim, S. C., Cook, J., and Bode, L. (2020). Testing the effectiveness of correction placement and type on instagram. The Inter. J. Press/Politics 25, 1940161220919082. doi:10.1177/1940161220919082

WHO World Health Organization (2020). DRAFT landscape of COVID-19 candidate vaccines-31 July 2020. Available at: https://www.who.int/ publications/m/item/draft-landscape-of-covid-19-candidate-vaccines (Accessed July 31, 2020)

Conflict of Interest: The authors declare that the research was conducted in the absence of any commercial or financial relationships that could be construed as a potential conflict of interest.

Copyright (c) 2021 Motta, Sylvester, Callaghan and Lunz-Trujillo. This is an openaccess article distributed under the terms of the Creative Commons Attribution License (CC BY). The use, distribution or reproduction in other forums is permitted, provided the original author(s) and the copyright owner(s) are credited and that the original publication in this journal is cited, in accordance with accepted academic practice. No use, distribution or reproduction is permitted which does not comply with these terms. 